\title{
Post-Cardiac Transplant Evaluation
}

National Cancer Institute

\section{Source}

National Cancer Institute. Post-Cardiac Transplant Evaluation. NCI Thesaurus. Code C100005.

A procedure to evaluate the health of the an individual after receiving a heart transplant. (ACC) 